\section{In tern ationa l}

Journal of

Medical

and Surgical Sciences

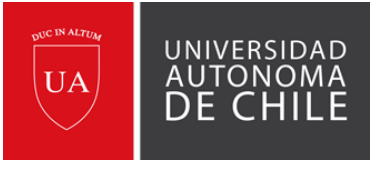

\title{
Idiopathic multicentric Castleman's disease, infrequent cause of Lupus-like. Case Report.
}

\author{
Juan Manuel Zapata, ${ }^{1}$ Fernando Andrés Lillo, ${ }^{1}$ Antonio Fabian Cabezas ${ }^{1}$ \&
} Santiago Felipe Andrés Riquelme. ${ }^{1}$

\section{ABSTRACT}

Castleman's disease (CD) or angiofollicular lymph node hyperplasia includes a heterogeneous mix of reactive lymphoproliferative processes with well-defined histological features. However, they differ in their localization patterns, clinical expression and etiopathogenesis. There are 4 types, one of them is the multicentric CD that is not associated with any viruses and has recently been called idiopathic MCD (iMCD). iMCD is a lymphoproliferative disorder with specific histopathological characteristics, more than one region of affected lymph nodes and absence of infection associated to human herpesvirus 8 and human immunodeficiency virus (HIV). iMCD covers multiple differential diagnoses and might simulate autoimmune diseases such as systemic lupus erythematosus. The aim of this article is to report the case of a patient with Castleman's disease and lupus-like presentation. We present the case of a 38-year-old man without morbid history, who presented lumbago, fever, diaphoresis and asthenia with two months of evolution, associated to bilateral cervical adenopathies. General examinations result negative, antinuclear antibodies at a dilution of 1/640 were positive, and extractable nuclear antigens were positive suggesting moderate Systemic Lupus Erythematosus (SLE) plus secondary Sjögren's. Methylprednisolone and Hydroxycloroquine boli were thus initiated. The patient evolved with anasarca, severe anemia, acidosis, polyserositis and multiple mediastinal adenopathies. Immunoglobulin and cyclophosphamide were thus initiated. He later presented fever, thrombocytopenia and nephrotic syndrome. Biopsy of cervical lymph node reported lymphadenitis with polyclonal plasmacytosis and concentric lymphoid hyperplasia, in agreement with iMCD. Treatment with Rituximab was initiated, which led to the favorable evolution of the patient. iMCD is a systemic inflammatory disease, its presentation corresponds to a constitutional syndrome resulting in a wide differential diagnosis. Every time suspicious adenopathies appear, they must be biopsied since this might lead to a definitive diagnosis.

Keywords: Giant lymph node hyperplasia, Interleukin-6, Systemic Lupus Erythematosus.

\section{INTRODUCTION}

Castleman's disease $(C D)$ or angiofollicular lymph node hyperplasia was described by Castleman et al. (1956) in a series of 13 cases with mediastinal masses mimicking thymomas. $C D$ includes a heterogeneous mix of reactive lymphoproliferative processes with well-defined histological features, which, however, differ in their localization patterns, clinical expression and etiopathogenesis. There are 4 types, each with a different diagnosis and treatment: 1) Unicentric CD (UCD); 2) Multicentric CD (MCD) associated to infection by human herpesvirus type 8 (HHV-8) and human immunodeficiency virus (HIV) (MCD-HHV8+/HIV+); 3) HHV-8-associated MCD, with no HIV association (MCDHHV8+/HIV-); and 4) MCD that is not associated to any of those viruses, which has been recently called idiopathic MCD (iMCD). CD presents one peak in young patients (30-40 years old) and another one around 60 years old, regardless of sex. The incidence of MCD-HHV8+/HIV+ has increased in the recent years as a product of AIDS' high incidence (García et al., 2016; Fajgenbaum, 2018).

rried out the entire case.

Acknowledgements: None.

doi: 10.32457/ijmss.2019.006.

iMCD is a lymphoproliferative disorder with specific histopathological characteristics, more than one region of affected lymph nodes and absence of infection associated to HHV-8 and HIV. There are multiple theories for iMCD's etiology, including infectious causes by agents not yet identified, autoimmune-based disease, germline 
mutations affecting innate immunity, or even neoplasias (Fajgenbaum et al., 2014). CD's prevalence in general, and in particular iMCD's prevalence, is unknown, it covers multiple differential diagnoses and might simulate autoimmune diseases such as Systemic Lupus Erythematosus (SLE) (García et al., 2016).

The aim of this article is to report the case of a patient with idiopathic multicentric Castleman's disease and lupus-like presentation.

\section{CASE PRESENTATION}

We present the case of a 38-year-old man without morbid history, who presented lumbago, fever, diaphoresis and asthenia with 2 months of evolution, associated to bilateral cervical adenopathies.

Laboratory tests revealed anemia, thrombocytopenia, hypoalbuminemia, acute renal failure (ARF) and erythrocyte sedimentation rate of $60 \mathrm{~mm} / \mathrm{h}$. Chest radiograph showed bilateral pleural effusion (Figure 1).

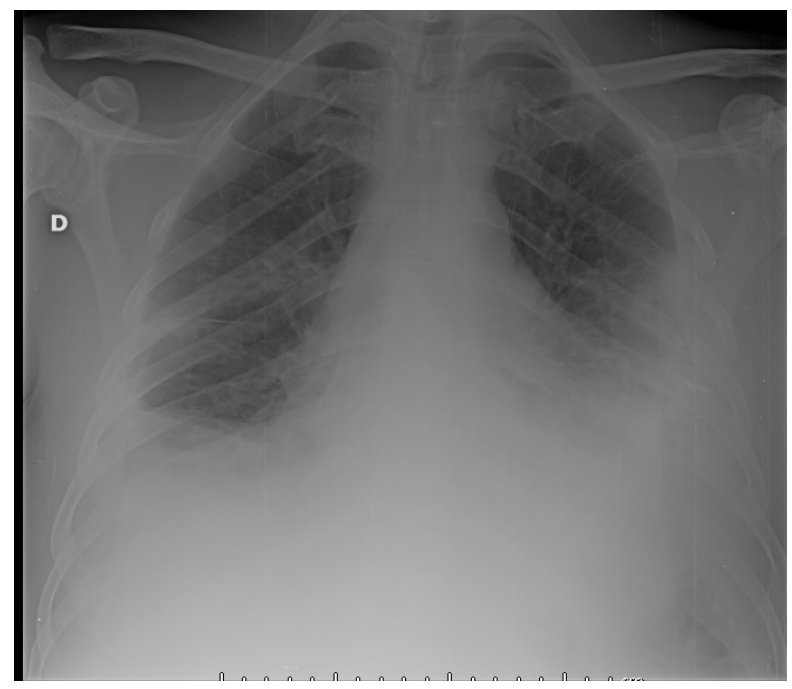

Figure 1. Chest radiograph showed bilateral pleural effusion.

HIV and HHV tests were negative, antinuclear antibodies at a dilution of $1 / 640$ were positive, extractable nuclear antigens were positive suggesting Sjögren's.

Methylprednisolone and Hydroxycloroquine boli were initiated due to Moderate SLE plus secondary Sjögren's.

The patient evolved with anasarca, severe anemia, acidosis, polyserositis and multiple mediastinal adenopathies (Figure 2). Immunoglobulin and cyclophosphamide were thus initiated. He later presented fever, thrombocytopenia and nephrotic syndrome.

Urine and blood cultures, as well as molecular analysis, were performed for bronchoalveolar lavage. All results were negative.

Biopsy of cervical lymph node reported lymphadenitis with polyclonal plasmacytosis and concentric lymphoid hyperplasia. No granulomas, fibrinoid necrosis, caseous necrosis or lymphoma components were found on the sample. Immunohistochemistry study was positive for CD138, and polyclonal kappa and lambda; CD3 and CD20 were positive in T and B lymphocytes. This proved that plasma cells that are widely present on the node mean no restriction for kappa and lambda chains, i.e., are polyclonal. This fact, as well as concentric hyperplasia, despite the lack of more solid vascular type structures (lollipop-type), might correspond to Castleman's lymphadenopathy of the polyclonal plasma cell variant (Figures 3, 4 and 5).

Treatment with Rituximab was initiated, which led to the favorable evolution of the patient.

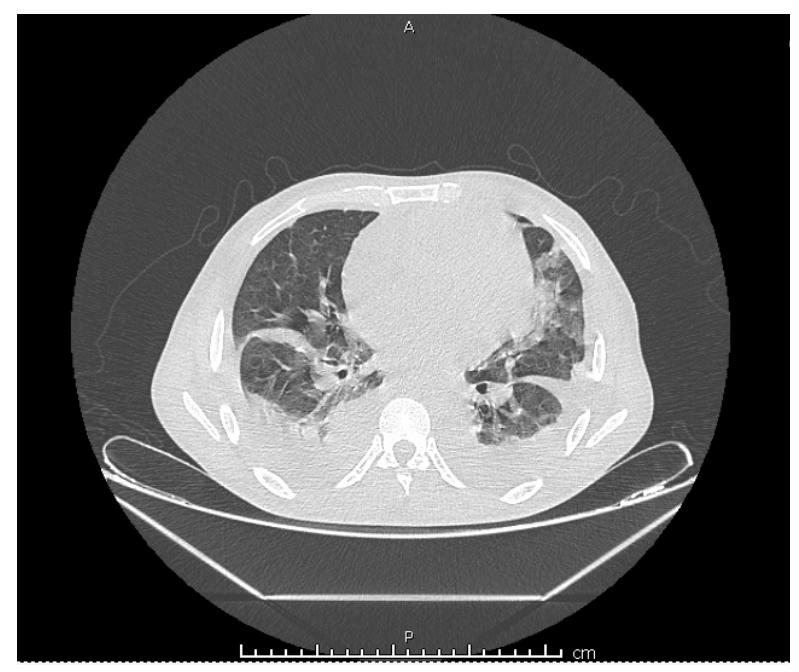

Figure 2. Pretracheal mediastinal adenopathies in aortopulmonary window measuring up to $13 \mathrm{~mm}$ in short axis. Additionally, bilateral axilary adenopathies are observed.

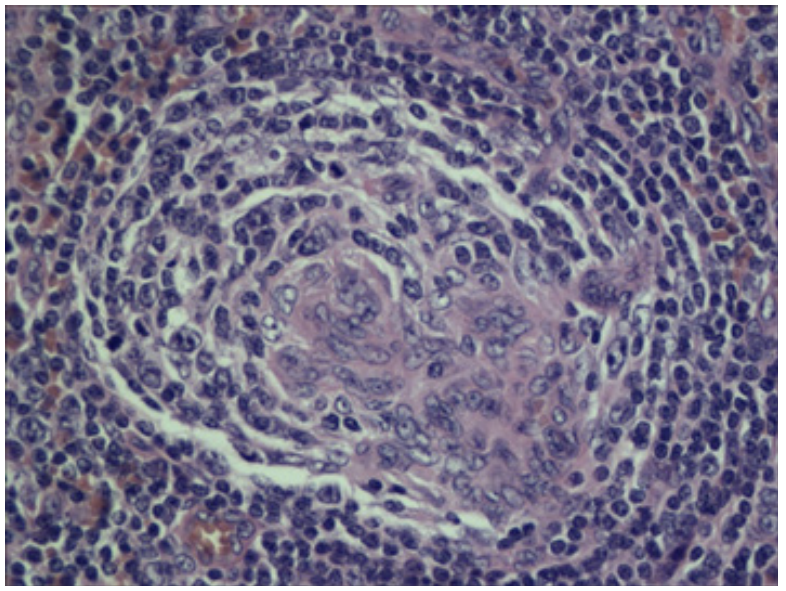

Figure 3. H-E staining: Lymphadenitis with polyclonal plasmacytosis and concentric lymphoid hyperplasia. 


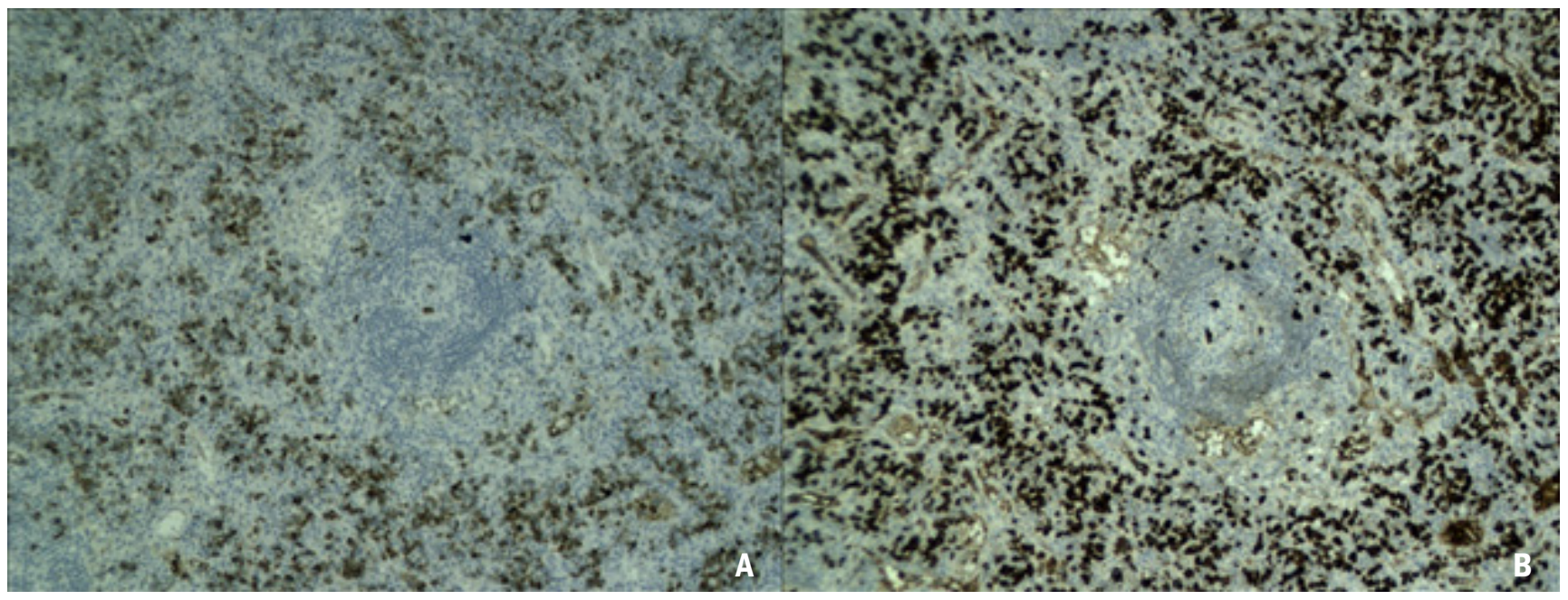

Figure 4. Immunohistochemistry: Positive for kappa (a) and lambda (b), revealing polyclonality.

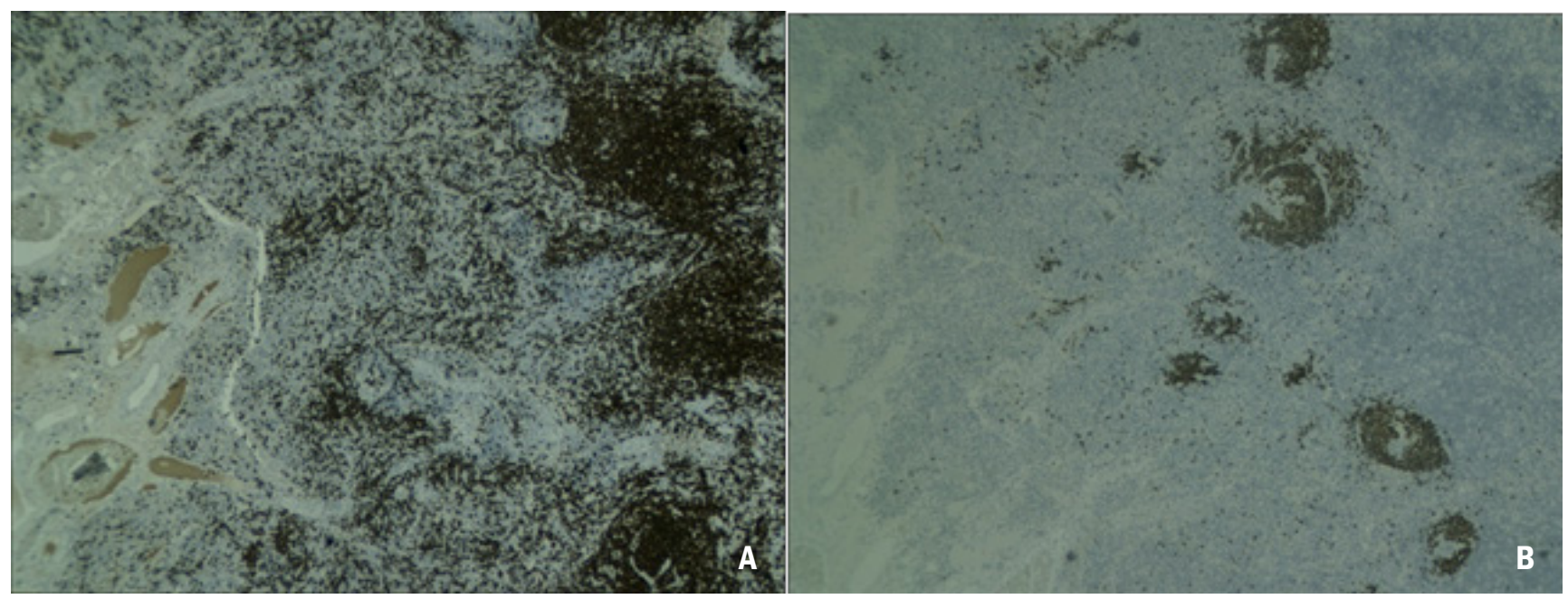

Figure 5. IImmunohistochemistry: CD3 (a) and CD20 (b) positive in T and B lymphocytes.

\section{DISCUSSION}

iMCD's etiopathogenesis is focused on inflammatory activity mediated by interleukin-6 (IL-6). It is manifested as a multi-systemic inflammatory disease that shares similar clinical elements with SLE. The recommended treatment is antibodies against IL- 6 or its receptor and in combination with other drugs in severe cases with ARF, severe anemia and serositis, as in the reported case. However, it must be noted that anti IL-6 therapy is not effective in all patients (García et al., 2016).

The clinical presentation will vary according to the disease's extension, blood cytokine concentrations and presence of HIV and HHV-8. In most of the cases, such as the reported, patients present prominent general symptomatology (fever, hyporexia, night sweats, asthenia) associated to the symptomatology of node or visceral affectation (Herrada et al., 1998). The association between SLE and iMCD has been documented in some cases reported in the literature, although in most of them,
iMCD diagnosis prevails (Shariatpanahi et al., 2016; Tokunaga et al., 2018; Wang et al., 2018; Zhang et al., 2016).

As a summary, iMCD is a systemic inflammatory disease, its presentation corresponds to a constitutional syndrome resulting in a wide differential diagnosis. Every time suspicious adenopathies appear, they must be biopsied since this might lead to a definitive diagnosis.

\section{REFERENCES}

Castleman B, Iverson L, Menendez VP. Localized mediastinal lymphnode hyperplasia resembling thymoma. Cancer 1956;9:822-30.

Fajgenbaum DC. Novel insights and therapeutic approaches in idiopathic multicentric Castleman disease. Hematology Am Soc Hematol Educ Program. 2018;2018(1):318-325.

Fajgenbaum DC, van Rhee F, Nabel CS. HHV-8-negative, idiopathic multicentric Castleman disease: Novel insights into biology, pathogenesis, and therapy. Blood 2014;123:2924-33. 
García AG, Cobo MM, de la Pena JP. Diagnóstico y tratamiento actual de la enfermedad de Castleman. Rev Clin Española 2016;216(3):146-156.

Herrada J, Cabanillas F, Rice L, Manning J, Pugh W. The clinical behavior of localized and multicentric Castleman disease. Ann Intern Med. 1998;128:657-62.

Shariatpanahi S, Pourfarzam S, Gheini M. Association of Macrophage Activating Syndrome with Castleman's Syndrome in Systemic Lupus Erythematosus. Iran J Pathol. 2016;11(3):265-271.

Tokunaga M, Yamada M, Yoshikawa S, Kondo A, Mishima M, Inoue S, Morita T, Tominaga N. Systemic lupus erythematosus with marked eosinophilia and clinical features mimicking TAFRO syndrome. Rinsho Ketsueki. 2018;59(6):688-694.

Wang L, Chen H, Shi J, Tang H, Li H, Zheng W, Zhang F. Castleman disease mimicking systemic lupus erythematosus: A case report. Medicine (Baltimore). 2018;97(38):e12291.

Zhang L, Cao XX, Wang SJ, Zhou DB, Li J. Clinical Features of Patients with Castleman's Disease Complicated Systemic Lupus Erythematosus. Zhongguo Yi Xue Ke Xue Yuan Xue Bao. 2016;38(5):543-547. 